\title{
Was bei alten COPD-Patienten anders ist
}

\author{
Mehr als die Hälfte der COPD-Patienten weist vier \\ oder mehr Begleiterkrankungen auf, überwiegend im \\ kardiometabolischen Bereich. Gerade bei alten und \\ sehr alten Patienten spielen daneben auch kognitive \\ Einschränkungen und Gebrechlichkeit eine wichtige \\ Rolle. Dem muss der Arzt diagnostisch und therapeu- \\ tisch Rechnung tragen.
}

_ Studien zur COPD spielen sich in der Regel in Kollektiven zwischen 60 und knapp 70 Jahren ab, wie Dr. Manfred Gogol, Krankenhaus Lindenbrunn in Coppenbrügge, ausführte. Doch erst bei noch älteren Patienten wird die Multimorbidität wirklich zum Problem. Deren Auswirkungen, z. B. auf die Progression der Erkrankung und die Effektivität der Therapie, sind somit gar nicht untersucht.

Neben kardiometabolischen Begleiterkrankungen spielen eine Abnahme der Muskulatur, Osteoporose und Depression/Angst sowie kognitive Dysfunktion eine wichtige Rolle für das Voranschreiten der funktionellen Einschränkung von COPD-Patienten.

\section{Kognitive Defizite berücksichtigen}

Je älter die Patienten sind und je mehr kognitive Defizite sie aufweisen, desto weniger aussagefähig wird auch die Messung der Lungenfunktion. Deshalb wird es immer schwieriger, auf dieser Basis therapeutische Entscheidungen zu treffen. Gut anwendbar sind jedoch auch bei kognitiv eingeschränkten Menschen ein 6-Minuten-Gehtest oder die Messung der Gehgeschwindigkeit, um den Funktionsstatus zu beurteilen. Auch die Handgreifkraft korreliert mit dem Peak flow.

Generell erhalten Patienten mit Demenz oder kognitiver Einschränkung weniger medizinische Leistungen als kognitiv gesunde Gleichaltrige. Das gilt auch für COPD-Patienten, wie Untersuchungen zeigen. Sie werden signifikant weniger behandelt als COPD-Patienten ohne Demenz.

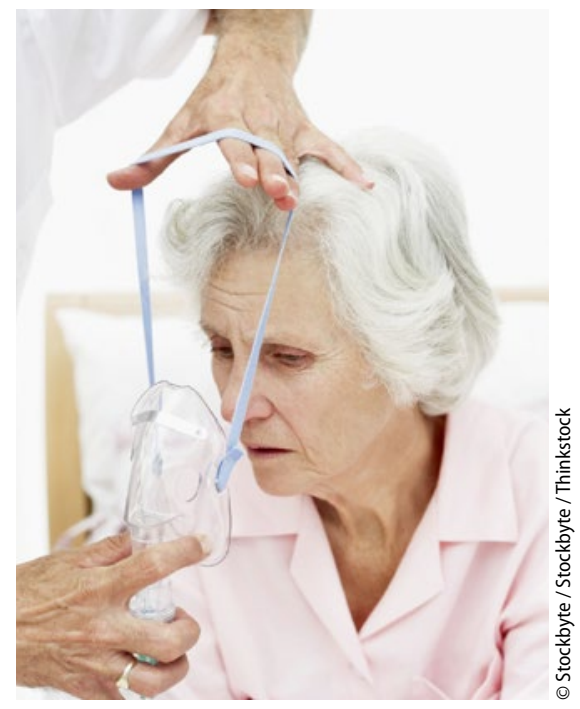

Gebrechlichkeit verdüstert die Prognose hochbetagter COPD-Patienten.

\section{Muskeln kräftigen}

Muskelschwäche ist eine wichtige Konsequenz der COPD, der frühzeitig entgegen gewirkt werden muss, z. B. durch Training oder neuromuskuläre Stimulation. Wegen der muskulären Probleme stellt die COPD auch einen wichtigen Risikofaktor für Stürze dar.

Sehr fragile COPD-Patienten, bei denen man keine Lungenfunktion mehr messen kann, sollte man trotzdem konsequent behandeln, wenn sie ein ausgeprägtes Emphysem aufweisen. Dosieraerosole sind für diese Menschen jedoch besser geeignet als Pulverinhalatoren. Denn die Kraft reicht nicht, um einen Pulverinhalator korrekt auszulösen.

$$
\text { Dr. med. Angelika Bischoff }
$$

- Symposium „Der multimorbide Patient", DGIM-Kongress Mannheim, 12. April 2016 